\title{
HapticArmrest: \\ Remote Tactile Feedback on Touch Surfaces Using Combined Actuators
}

\author{
Hendrik Richter, Sebastian Löhmann, Alexander Wiethoff \\ University of Munich, Germany \\ \{hendrik.richter, sebastian.loehmann, \\ alexander.wiethoff\} @ifi.lmu.de
}

\begin{abstract}
Interactive surfaces form an integral component of intelligent environments. In the paper, we describe HapticArmrest, a simple tactile interface that communicates tactual surface characteristic and form of interactive elements on direct touch surfaces. Spatially separating manual touch input and active tactile output allows for the combination of various types of tactile actuators for versatile haptic feedback. In a preliminary experiment, we indicate that our approach enables a reliable discrimination of virtual elements on touch surfaces solely based on tactile representations. We also assessed the hedonic and pragmatic qualities of the generated tactile stimuli by applying methods from the field of usability research.
\end{abstract}

Keywords: interactive surfaces, haptics, tactile feedback, touch, actuators

\section{Introduction}

Due to advances in display technology and sensing devices, surfaces responsive to direct manual touch are all around. The flexibility of touch-based graphical user interfaces allow for devices in a multitude of sizes and forms (mobile phones, tablet PCs, tabletops, public displays etc.). Interactive surfaces are used in dynamic scenarios that could involve personal and public use, noise or high visual and cognitive load. Despite the ubiquity of touch input and the benefits of multimodal signals in ubiquitous scenarios, most touch interfaces still lack tactile output. Touch screens only present a flat, uniform surface to the interacting user's hand; all GUI elements provide the same reduced cutaneous (i.e. tactile) impression.

Scientific approaches to provide versatile tactile sensations to the human skin are in existence. Evaluations show that users of touch surfaces greatly benefit from tactile feedback. This is true for both objective measures and emotional aspects of the interaction $[10,15,5,20]$. Still, haptic sensations are underused as a redundant information channel in the fields of mobile interaction or pervasive and physical computing [24]. A possible reason is a lack of haptic signal generators that are versatile and effective, but at the same time small and non-expensive [8]. Current high-bandwidth solutions mostly entail technical complexity and space issues. A potentially high number of bulky, complex and power consuming tactile actuators have to be implemented into the touch interface (see part 3). This impedes the application of tactile cues as an additional channel of information in ubiquitous scenarios. 

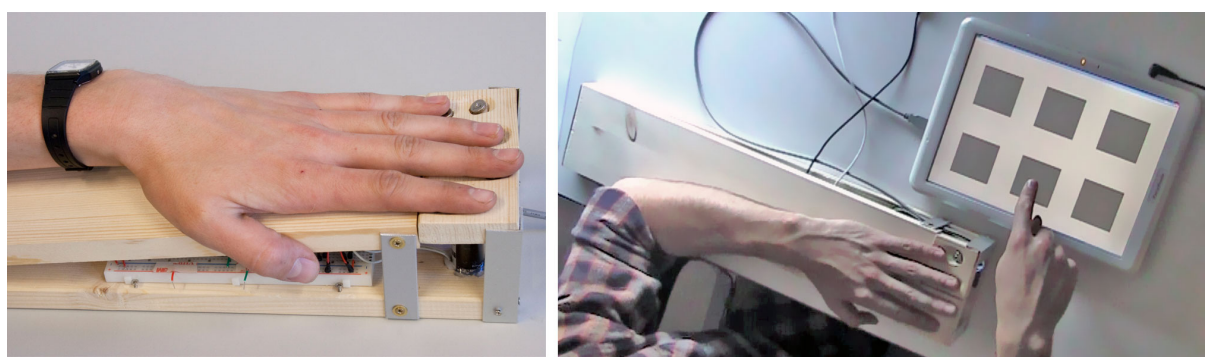

Figure 1: The HapticArmrest (left) communicates tactile patterns and movements to the user's non-dominant hand. When touching an interactive element with the opposite hand, the user feels the form and surface characteristics of this item (right).

To cope with these challenges, we propose the spatial separation of manual touch and resulting tactile feedback. By doing so, we may be able to combine cheap and simple haptic actuators for nonetheless meaningful and rich tactile feedback on touch interfaces. Additionally, our approach may help to reduce the complexity and size of used actuators and is applicable on arbitrary interactive surfaces. We built a prototypical haptic interface (see Figure 1) with dual type touch feedback by combining different actuators. Thus, we provide both haptic (movement of fingers) and tactile (vibrations on the skin) sensations. In a first evaluation (see part 5.1), we show that users are able to reliably discriminate visually identical interactive elements solely depending on the generated remote tactile stimuli. Additionally, in order to assess the hedonic and pragmatic qualities of the conveyed tactile stimuli, we applied methods from the field of usability research (see part 5.2). Here, the users indicate the livening and positive nature of the designed stimuli and give rise to improvements of the prototype.

\section{Remote Tactile Feedback and Combined Actuators}

As stated before, we spatially separate manual touch input and resulting tactile output. We believe that this approach of having remote tactile feedback provides three main consequences:

(1) We can combine actuators that differ in size, complexity and stimulus characteristics.

(2) We can produce combined tactile feedback for the communication of meaningful and rich content such as form and abstract state of interactive elements.

(3) Any surfaces can be augmented with remote tactile impressions as the surface itself does not have to be modified; the electromechanical infrastructure is not restricted to the size and form of the surface.

In order to assess the feasibility of our approach, we designed a prototypical tactile interface, the HapticArmrest (see Figure 1). Our prototype comprises two types of actuators:

a) Eccentric Motors: This low-complexity type of actuator generates structured vibrotactile patterns that can be dynamically modified by altering signal parameters such as amplitude, frequency or rhythm. The user perceives the stimuli as rhythmic vibrations on the skin. 
b) Linear Solenoids: This off-the shelf component communicates highly dynamic stimuli such as changes of pressure or movement. The user recognizes a sudden change of height of the object under his fingertips.

Feedback occurs in three different situations. First, the edges of an object can be touched directly. Second, the user can slide his finger onto a virtual object (rollover) or leave the object (rollout), which includes cutting across an edge. Third, surface textures of virtual objects can be explored. This system behavior is based on common state models to describe interactions with direct manipulation interfaces [4]. Technical details on actuators and stimulus design are specified in part 4.

Instead of augmenting the user with wearable technology, we decided to instrument the user's direct environment. In this case, it is possible for the user to lift his arm from the device to use both hands for the interaction or to stop exploring the surface. When thinking of scenarios such as office areas or cars in which the users tend to remain in direct contact with chairs, seats or tables, it is easier to implement the actuator technology in the user's direct environment. For scenarios involving interactive tables or walls, remote tactile feedback may even be conveyed by actuators in the frame of the device or even in the floor. In this case, every single user may feel distinct and personal tactile stimuli that is responsive to the specific context of the user. Tactile actuators similar to our prototype may be implemented in a frame of a public interactive table and could communicate characteristics of virtual elements and acknowledgements for activations in order to reduce visual load.

We tested the basic potential of our approach in a preliminary experiment. Our interest was twofold. We wanted to assess if people are able to use the additional channel of tactile information on touch surfaces despite the spatial separation of manual action and resulting stimulus. Therefore, we designed a setup in which participants had to identify interactive GUI-elements solely based on tactile information. Furthermore, we wanted to look at the usability and hedonic quality of the tactile stimuli we created by combining sensations from the motors and solenoids. Methods for the evaluation of tactile stimuli are sparse [14]. Therefore, we transferred methods from usability research such as AttrakDiff [9] for the comparison and summative evaluation of the two different stimuli.

\section{Related Work}

Thanks to technological advances in the development of interfaces (processing power, sensors, actuators), the interest in the use of multimodal information processing is constantly growing [18]. Multimodal interaction is beneficial in highly dynamic scenarios with environmental factors such as noise, vibration or narrowness [7]. The redundant use of multiple senses helps to improve the interaction as a whole [11]. With touch interfaces being the most commonly used interfaces in dynamic scenarios, the use of haptics comes into play.

The communication of actively generated tactile information to the human's skin has been studied for a long time in fields such as virtual reality, accessibility and Sensory Substitution [8]. Researchers utilize artificial haptic stimuli to transmit either abstract information such as warnings, acknowledgements or system states or 
palpable characteristics of virtual elements such as form or malleability onto the user's skin $[8,16]$.

Interfaces that provide tactile feedback for direct touch mostly fall into one of three categories: First, miniature actuator system such as [19] move the mobile device or the device's screen as a whole. Thereby only a single point of touch input can be supported by tactile stimuli. The approach does not scale for tabletops or interactive walls. Systems such as Tesla Touch [2] convey tactile signals based on electrical stimuli, but also only provide single touch input. Second, a form of tactile interface that is used for a long time is a tactile display such as FEELEX [12]. The interactive surface is segmented into individually movable 'tactile pixels'. Currently, this approach results in a reduced tactile and visual resolution due to mechanical complexity and size of the individual actuators. Third, tangible user interfaces atop the interactive surface such as [16] could help to communicate versatile stimuli using various types of actuators. All of these approaches postulate in essence that tactile feedback for an interaction should be applied directly to the body part that is in contact with the screen, i.e. mostly the fingertip.

By contrast, we try to evaluate the feasibility of remote tactile feedback. Few researchers have incorporated distal tactile stimuli before [21]. However, they presented promising impacts on interaction speed and text entry [17]. The more general approach of tactile sensory relocation has been researched and used before in the fields of accessibility and Sensory Substitution [1]. For example, Clippinger et al. [6] describe a sensory feedback system for an upper-limb amputation prosthesis. Here, sensors in the gripper-like prosthesis capture the level of closing pressure. This information is communicated to remote parts of the user's body using electrotactile actuators. This approach of tactile sensory relocation helps the user to precisely control the force used for grasp and pinch with the artificial hand.

\section{HapticArmrest: Technical Details}

The decision to design an armrest was influenced by an observation made by Ryall et al. [22]. When interacting with direct-touch tabletops, people tend to lean on the surface with their non-active hand or arm. This leads to accidental inputs and thus to confusing reactions of the system. Haptic Armrest offers a placement area for the user's idle arm. 

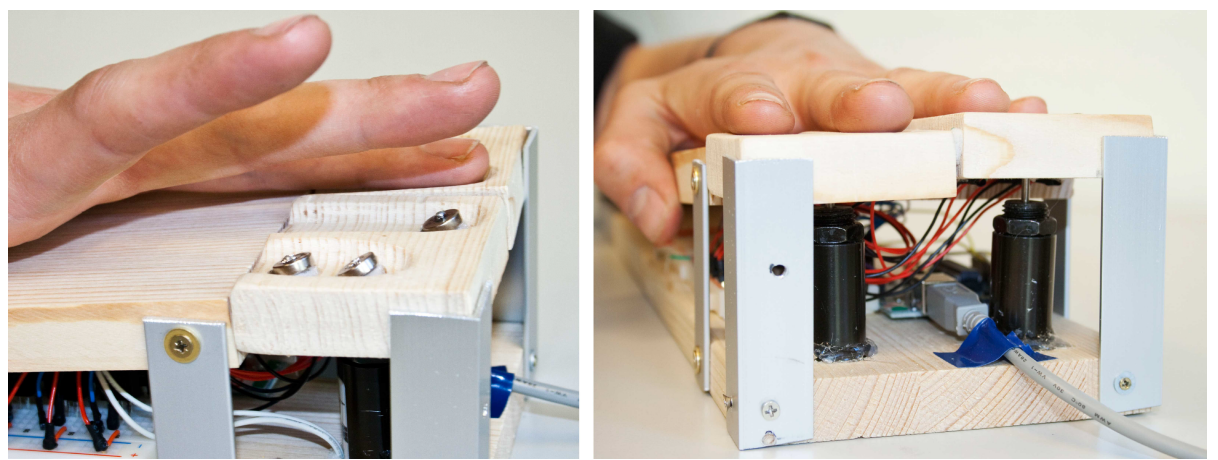

Figure 2: View of the two types of actuators on the HapticArmrest. Eccentric motors (left) and linear solenoids (right).

\subsection{Actuators}

HapticArmrest incorporates two types of actuators (see Figure 2). Solenoids provide haptic feedback by lifting the user's fingers, while vibration motors, commonly used to shake mobile phones, apply vibrotactile stimuli to the fingertips. We used the number of single actuators of every type as an additional parameter to design the abstract stimuli.

\section{Eccentric Motors.}

As the prototype can be used by both left- and right-handed persons, altogether six vibration motors have been installed. Four of them provide feedback to all fingers of the right hand besides the thumb. Because of the different length of little and index finger, two more motors are needed for left-handed users. Each motor can be triggered separately, which allows for a stimulation of an arbitrary number of fingertips at the same time. This kind of tactile feedback can be used to simulate the exploration of an object's surface texture. These textures do not resemble materials of the real world, but are designed for articulate distinction.

\section{Solenoids.}

Each of the two built-in solenoids is connected to a wooden pad, which can be lifted by up to four millimeters. Each pad includes two cavities giving the user a hint where to place the fingers. This kind of feedback was used during the evaluation to simulate the touch of an object's edges, including rollover and rollout.

\subsection{Signals}

For both types of feedback, so called tactons (tactile icons) [3] where used to create different feedback patterns. The varying parameters were rhythm, duration and the combination of different actuators. Considering the vibration motors, three rhythms were used: permanent vibrations, alternating activation and deactivation of the motors and, what we called "wave", activating the four motors one after another, starting with the small finger. The duration of the stimuli was either 200 or 400 milliseconds 
[13]. The vibrations were applied to the small finger, the index finger or all fingers besides the thumb. Given these parameters, we created twelve different feedback patterns. We followed the same procedure with the two solenoids by lifting different fingers with a certain duration and rhythm.

The combination of both types of haptic feedback, vibrations and lifting the fingers, allow for a fourth parameter and thus for the creation of new patterns and possibly even new haptic stimuli.

\section{Evaluations}

With our evaluation, we wanted to verify that users are able to utilize the remote tactile stimuli as an additional and synchronous channel of information when manipulating and exploring interactive elements. Therefore, we designed a task in which participants had to discriminate virtual elements using tactile signals created by the EdgeMatrix. Touch feedback can have a strong emotional impact [23], so we tried to assess hedonic quality and usability of our signals and the system by applying standard measures from the field of usability research (see part 5.2).

\subsection{Discrimination Task}

The experimental setting is depicted in Figure 1. We conducted the experiment using a touch based tablet $\mathrm{PC}$ which depicted 6 gray squares $(4 \times 4 \mathrm{~cm})$. A touch on a square resulted in two types of feedback (edges, areas) generated by the HapticArmrest (see part 4). During each test trial, two of the six elements shared a common tactile characteristic.

Twelve volunteers ( 6 female), ranging in age from 20 to 27, were recruited for the experiment. All participants were right handed. They wore earmuffs to isolate environmental noise. After an introduction and a training phase, each participant completed six trials. During a trial, the participant was free to touch and explore the depicted elements. He was asked to point out to the pair of elements with the same tactile impression. In three of the six trials, a pair of elements with identical tactile 'surface-impression' was depicted. In the other three trials, a pair of elements with identical tactile 'edge-impression' had to be identified. Elements that were not part of the pair had randomly selected tactile characteristics. The order of the tactile representation was counterbalanced. Each tactile pattern representing a pair of elements was only presented once for every participant. In total over all participants, 72 pairs of GUI elements had to be identified (36 for each actuator technology). 


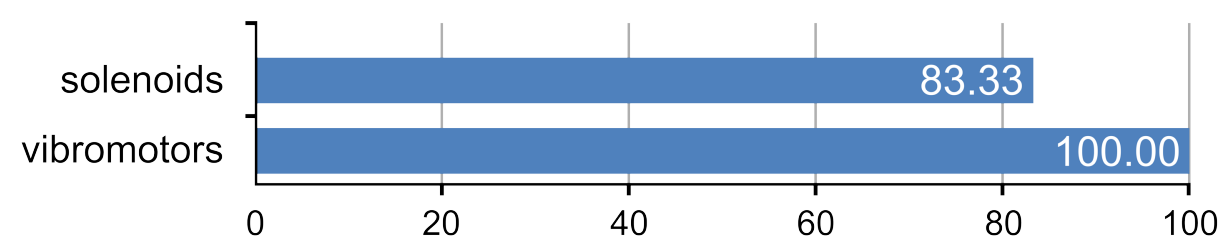

Figure 3: Percentage of correctly identified pairs of tactile GUI elements for each actuator technology (solenoids $=$ edge feedback, vibromotors $=$ surface feedback)

Results.

The results in Figure 3 show that all pairs of elements with identical remote vibrotactile feel could be identified by the participants. For the pairs of elements with tactile edges using vertically moving solenoid actuators, in six out of 36 trials participants were not able to identify one pair of elements out of three. This results in a discrimination rate of 83.33 percent for the solenoid actuator technology.

\subsection{Hedonic and Pragmatic Qualities}

As this type of feedback modality is still emerging from the research environment we also investigated the hedonic qualities users connected with the feedback. Therefore we conducted an evaluation based on AttracDiff [9] by Hassenzahl et al. This scientific method is targeted towards revealing additional to the general usability and performance of the system the emotional and hedonic quality of an interaction modality. Pairs of opposing adjectives (semantic differentials) are presented to the participant who is asked to rate the system based on this measure on a scale from -3 to +3 . We tested and evaluated two signal types: (a) vibrotactile stimuli, represented in Figure 4 through squares and (b) pressure plus movement indicated in figure 4 with circles.

The most noticeable results from the test indicated that both feedback modalities were perceived as rather technical (mean=-1.0) vs. human by the users (see Figure 4). Further, the approach of providing such feedback signal types was recognized as being quite creative (mean=2.0) compared to uninspired on the other side of the spectrum. A difference between the approaches was stated regarding the feedback type (a), which led to the result of being pleasant compared to rather unpleasant (mean=0.5) for feedback type (b) with a mean of 1.5.

In general, the closeness of the results of both signal types (see figure 4) can be interpreted in a way that the participants did not perceive the two stimuli as completely separate feedback types. This supports our assumption that we can convey tactile stimuli resulting from multiple combined remote actuators. Regarding the technicality of our approach, we will further substantiate this issue in future case studies by applying different immaterialities to the housing of the actuator technology (e.g. organic user interfaces). The general pleasance and perceived creative nature of both approaches seem worthwhile investigating, for example by applying different actuator technologies, thus increasing the pleasance of use. 


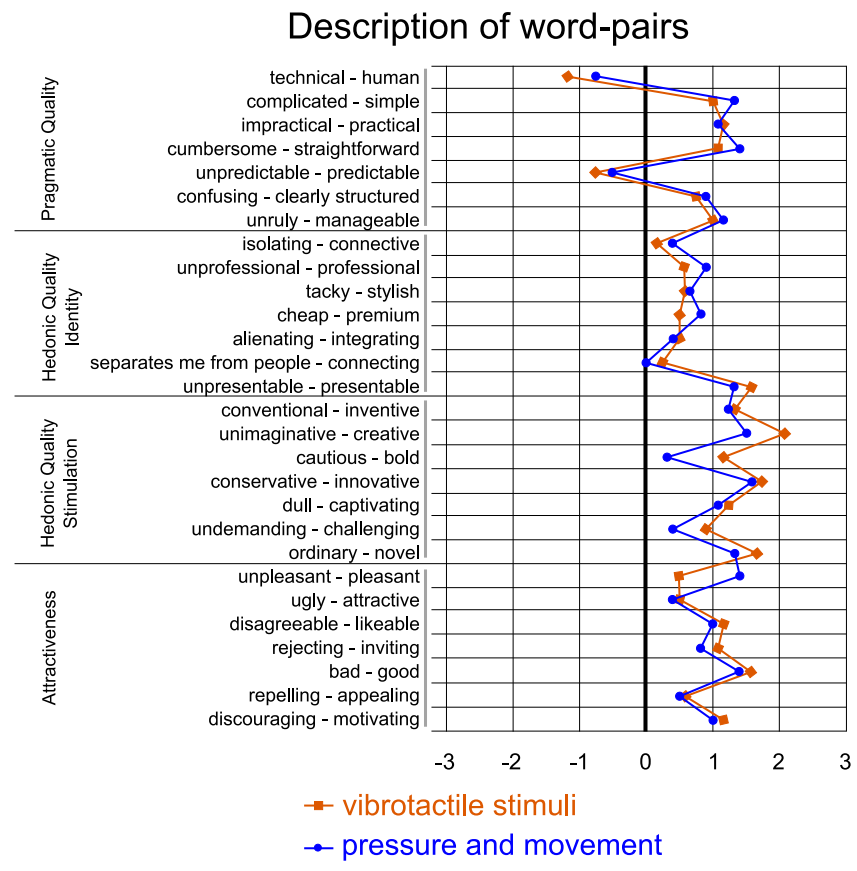

Figure 4: Results of AttrakDiff

\section{Discussion}

Considering the results of both studies, we can state the following: The HapticArmrest has been a valuable device for the exploration of the potential of remote tactile feedback and the combination of tactile stimuli. We used low-cost and low-complexity actuators to communicate two different types of stimuli to haptically augment interactive graphical elements. Yet, we did not measure the influence of noise and (subjectively unnoticeable) latency produced by our off-the shelf components. Still, due to their reduced complexity, the actuators may easily be implemented in tactile interfaces and the user's direct environment. Furthermore, our results indicate that it is possible for the user to reliably identify interactive elements based on stimuli that are created by this type of low-complexity actuators.

The distinctive feature of our approach is the spatial separation of touch-based input and artificial tactile output. Thus, any surface might be augmented with remote tactile feedback. Our results indicate that even with the remote application of tactile stimuli, the simultaneous feedback is still understandable and usable during an interaction.

Due to the aforementioned impact of tactile stimuli on the human emotion system, we also have to evaluate the usability and hedonic quality of the tactile stimuli designed in this way. Quantitative empirical evaluations for the analysis of tactile perception across the body surface or the effects of tactile feedback on error rates are well established (e.g. in [10]). In contrast, we lack standard methods to evaluate 
qualitative aspects of artificial tactile stimuli. Such methods could help to repeat and validate our investigations. For a start, we used methods from the field of usability research such as AttrakDiff (see part 5.2).

With AttrakDiff, the participants rated the stimuli based on semantic differentials, i.e. pairs of opposing adjectives (see Figure 4). However, some of these pairs (such as cautious vs. bold) do not quite fit. Participants stated their difficulties to use these adjectives to describe emotional or perceived aspects of artificial sensations to touch. However, word-pairs such as cheap vs. premium or pleasant vs. unpleasant do cover tactile feedback well and enable us to improve the design of future tactile interfaces and stimuli. In general, the most distinctive results can be found in the sections Hedonic Quality - Stimulation and Attractiveness. For future evaluations, we plan to extend these sections in order to cover the special characteristics of tactile stimuli. A more customized evaluation method covering the hedonic qualities of non-visual stimuli could give rise to the design of future interfaces.

\section{Conclusion and Future Work}

With the HapticArmrest we introduced an inexpensive hardware design for a device that allows for the exploration of remote tactile feedback on touch surfaces. Our prototype incorporates cheap and simple off-the-shelf actuators such as vibrational motors and linear solenoids. We applied sudden movement and diffuse vibrotactile stimuli to express both form and surface characteristics of virtual buttonlike screen elements. In a preliminary experiment, we explore the effectiveness and usability of remotely applied feedback.

In the future, we intend to further advance our user interface. We already received valuable comments that suggest to reduce the noise or to alter the form of our interface. By using smaller and less conspicuous actuators, our interface could blend into the user's direct environment. We also think about using other locations of the user's body to place the actuators. The areas of the human skin differ in two-point, frequency and amplitude discrimination thresholds. Thus, more pressure would be needed to create the same amount of tactile stimulation. However, large usable areas such as the human back lend itself for the application of tactile stimuli. Adding other somatosensoric modalities such as the perception of heat or moisture by using appropriate actuators could further enrich and improve the interaction.

In summary, our work questions the assumption that tactile feedback has to be given at the interacting fingertip or hand. With remote tactile feedback, we can augment touch surfaces no matter what technology, form or size. The integration of different forms of actuators creating tactile stimuli which complement one another may become possible. Novel forms of multimodal interfaces may help us in enhancing and enriching the dynamic interaction with ubiquitous and multimodal interfaces.

\section{References}

1. Bach-y-Rita, P., and W Kercel, S. Sensory substitution and the human-machine interface. Trends in cognitive sciences 7(12), pp. 541-6, (2003) 
2. Bau, O., Poupyrev, I., Israr, A., and Harrison, C. TeslaTouch. Proc. UIST '10, 283292, (2010)

3. Brewster, S. and Brown, M. Tactons: structured tactile messages for non-visual information display. Proc. AUIC '04, Vol. 28, 15-23, (2004)

4. Buxton, W., A three-state model of graphical input. Proc. INTERACT '90, NorthHolland Publishing, pp. 449-456, (1990)

5. Chang, A. and O'Sullivan, C. Audio-haptic feedback in mobile phones. Proc. CHI '05, pp. 1264-1267, (2005)

6. Clippinger, F., Avery, R., and Titus, B. A sensory feedback system for an upper-limb amputation prosthesis. Bulletin of prosthetics research, pp. 248-258, (1974)

7. Cohen, Philip R. and McGee, David R. Tangible multimodal interfaces for safetycritical applications. Communications of the ACM 47, pp. 41-46, (2004)

8. Gallace, A., Tan, H. Z. and Spence, C. The Body Surface as a Communication System: The State of the Art after 50 Years. Presence: Teleoperators \& Virtual Environments, 16(6), pp. 655-676, (2007)

9. Hassenzahl, M. and Tractinsky, N. , User experience - a research agenda. Behavior \& Information Technology, 25(2), pp. 91-97, (2006)

10. Hoggan, E., Brewster, S.A., and Johnston, J. Investigating the Effectiveness of Tactile Feedback for Mobile Touchscreens. Proc. CHI '08, pp. 1573-1582, (2008)

11. Hoggan, E., Crossan, A., Brewster, S. A., and Kaaresoja, T. Audio or tactile feedback: which modality when. Proc. CHI '09, pp. 2253-2256, (2009)

12. Iwata, H., Yano, H., Nakaizumi, F., and Kawamura, R. Project FEELEX: adding haptic surface to graphics. Proc. SIGGRAPH '01, pp. 469-476, (2001)

13. Kaaresoja, T. and Linjama, J. Perception of short tactile pulses generated by a vibration motor in a mobile phone. Proc. EuroHaptics '05, pp. 471-472, (2005)

14. Koskinen, E., Kaaresoja, T., and Laitinen, P. Feel-good touch. Proc. IMCI '08, pp. 297-304, (2008)

15. Leung, R., MacLean, K., Bertelsen, M.B., and Saubhasik, M. Evaluation of Haptically Augmented Touchscreen GUI Elements under Cognitive Load. Proc. ICMI '07, pp. 374-381, (2007)

16. Marquardt, N., Nacenta, M., Young, J., Carpendale, S., Greenberg, S., and Sharlin, E. The Haptic Tabletop Puck: Tactile Feedback for Interactive Tabletops. Proc. ITS'09. pp. 93-100, (2009)

17. McAdam, C., and Brewster, S. Distal tactile feedback for text entry on tabletop computers. Proc. of BCS-HCI '09, pp. 504-511, (2009)

18. Oviatt, S. Multimodal interfaces. In J. A. Jacko and A. Sears (Eds.), The humancomputer interaction handbook, pp. 286-304, (2002)

19. Poupyrev, I., Maruyama, S., and Rekimoto, J. Ambient touch: designing tactile interfaces for handheld devices. Proc. UIST '02, pp. 51-60, (2002)

20. Richter, H., Ecker, R., Deisler, C., and Butz, A. HapTouch and the $2+1$ State Model Proc. AutomotiveUI '10, pp. 72-79, (2010)

21. Richter, H. Multi-Haptics and Personalized Tactile Feedback on Interactive Surfaces. EuroHaptics '10, (2010).

22. Ryall, K., Forlines, C., Shen, C., Morris, M. R., and Everitt, K. Experiences with and observations of direct-touch tabletops. Proc. TABLETOP '06, pp. 89-96, (2006)

23. Salminen, K., Surakka, V., Lylykangas, J., Raisamo, J., Saarinen, R., Raisamo, R., et al. Proc. CHI '08, pp. 1555-1562, (2008)

24. Wright, A. The touchy subject of haptics. Communications of the ACM, 54(1), pp. 20-22. (2011) 